\title{
LA GRANDE DAME DE LA PSYCHOLOGIE SOCIALE
}

\author{
Denise Jodelet \\ Ecole des Hautes Etudes en Sciences Sociales, Paris, França
}

La figure de Sílvia Lane s'identifie pour moi avec la découverte de ce que signifie une véritable ouverture de la psychologie sociale aux réalités du monde social. C'est elle aussi qui me fit pénétrer, il y a maintenant vingt cinq ans, après un premier contact avec le Nordeste, dans la vie de cette discipline dont elle a impulsé le développement au Brésil. Et c'est par son intermédiaire que j'ai noué d'étroites relations de travail avec certains de ses anciens élèves, notamment Celso Pereira de Sà de l'Université d'Etat de Rio de Janeiro, Clelia de Nacimento Schltze et Brigido Viezu Camargo de l'Université de Florianopolis.

Tout commença en 1982. Angela Arruda, aujourd'hui professeur à l'Université Fédérale de Rio de Janeiro, que j'avais orientée lors de ses études l'Ecole des Hautes Etudes en Sciences Sociales, m'invita à faire un cours dans l'université de Campina Grande où elle venait d'être nommée après un long exil. Ce qui fut l'occasion d'entamer des relations durables avec maints collègues du Nordeste. Je ne sais comment Sílvia avait appris ma venue au Brésil; en tout cas, elle me contacta pour participer à un symposium sur la psychologie sociale qu'elle organisait dans le cadre de la réunion de l'ASBPC à Campinas. Je répondis avec émotion à cette invitation inattendue et prometteuse.

Comme un vent de passion soufflait dans cette assemblée portée par l'élan de Sílvia, encore toute vibrante du périple qu'elle venait de faire, avec son amie et collègue Maria do Carmo, telles des missionnaires d'une authentique psychologie sociale, dans les divers pays d'Amérique Latine. Cet évènement fut pour moi une véritable révélation. Une double révélation: celle de ce que pouvait et devait être une psychologie sociale en prise avec les problèmes et les réalités de la vie sociale; objectif qui restait de l'ordre du voeu pieux dans la plupart des recherches de l'autre côté de l'Atlantique. Celle d'une réflexion qui associait l'ensemble des chercheurs appartenant à des pays marqués par un même destin historique. Bref une perspective historico-culturelle engagée éthiquement et politiquement qui devait, pour toujours, ouvrir des horizons à ma pratique de recherche et la conforter dans ses attendus. De là vient sans doute qu'il me fut si facile, par la suite, de rencontrer les intérêts et les engagements de mes collègues au Brésil et au-delà en Amérique Latine. Dès cet instant Sílvia Lane fut pour moi "la grande dame de la psychologie sociale" que je saluais à l'ouverture de la deuxième Conférence Internationale sur les Représentations Sociales qui s'est tenue à Rio de Janeiro en 1994.

Ce n'est pas tout. Le symposium de l'ASBPC permit à une réelle amitié de se nouer, et nous échangeâmes des visites entre Paris et Sao-Paulo. Proches par l'âge et la passion intellectuelle, nous nous sentions comme deux soeurs. Quelques soirées familiales, avec ses enfants et son vieux père, restent chères à mon souvenir. J'y ai découvert l'histoire de toute une vie d'espérances, de menaces, de résistances et aussi de malheur mal maîtrisé.

Sensible à mes intérêts, Sílvia a un temps porté, non sans esprit critique comme en témoignent ses publications, son attention sur les représentations sociales. Elle m'a aussi ouvert la porte de "terreiros" d'Ubanda que je désirais connaître. Elle était ouverte à tout. Nos chemins se sont ensuite séparés. Sílvia s'orientait vers la psychologie communautaire et j'étais invitée dans d'autres universités brésiliennes. Les nombreux séjours que j'avais fait au Département de Psychologie Sociale de la Pontifícia Universidade Católica (PUC) de Sao Paulo n'eurent pas de suite, alors qu'elle même devait s'en trouver écartée. Nos dernières rencontres remontent aux Congrès de Associação Brasileira de Psicologia Social (l'ABRAPSO), à Bel Horizonte et Sao-Paulo, à la fin des années 90 . Je conserve précieusement de ce dernier, une photo joyeuse où nous posons en riant avec Muné et Gonzales Rey; ce que j'appelais le dernier quarteron de psychologues sociaux matérialistes! Mon projet d'aller lui rendre visite lors d'un passage au Brésil en 2006, n'eut pas le temps de se réaliser: la nouvelle de sa mort s'abattit sur notre communauté quand j'étais encore à Brasilia.

Un des éléments qui m'a le plus frappé dans la personnalité de Sílvia est son indépendance et son immense ouverture d'esprit. Elle avait fait un son doctorat aux Etats-Unis, mais n'en gardait nulle dépendance intellectuelle. Et je me souviens d'une réflexion qu'elle fit à la suite d'un voyage en Europe. En effet, venant à Paris, elle en profita pour visiter quelques universités. L'Europe lui sembla, dit-elle à son retour, comme "un vieil et beau théâtre poussiéreux." Elle parlait d'un lieu, son pays, son continent, où tout est à faire et surtout à réinventer, contre l'oppression du passé et la douleur du 
présent. Et de fait, elle renouvelait tout ce qu'elle touchait. Un exemple: attentive au courant des représentations sociales auquel elle se familiarisait, sur le plan théorique et méthodologique, à mon contact, elle eut vite fait de n'en retenir que ce qui correspondait à ses préoccupations d'emprise sur les réalités concrètes de ses champs de travail. Ainsi, s'inspirant du modèle d'analyse structurale des représentations sociales, conçut-elle un schéma d'extraction des réseaux sémantiques qui structurent des discours recueillis au cours d'entretiens non directifs. Elle associait ainsi au désir d'exploiter des propositions d'approches nouvelles, sa fidélité aux méthodes qualitatives qui permettent une saisie authentique de l'expérience de sujets inscrits dans leur milieu de vie, et son attention pionnière au langage. Elle donnait aussi la preuve d'une autre de ses grandes qualités: l'inventivité. Sílvia était une véritable chercheuse: elle savait trouver.

Car, il faut aussi souligner combien Sílvia Lane a devancé par ses propositions bien des tendances qui prennent aujourd'hui de l'importance dans la psychologie sociale. Pour n'en citer que quelques unes, mentionnons ses réflexions sur les rapports entre le langage et la pensée ou le langage et l'action; sa centration sur les processus idéologiques qui infléchissent les discours sociaux ou les positions individuelles; et surtout sa contribution à l'étude des émotions où elle devança bien des auteurs tout en restituant sa place à l'apport de Vigotsky. Dans tous ces domaines, elle a fait oeuvre de théoricienne, tout à la fois innovante et soucieuse de maintenir dans sa ligne d'horizon des phénomènes qui, comme l'idéologie, semblent à certains relever de préoccupations obsolètes. Cette oeuvre de théoricienne, parfaitement adaptée à la saisie de la complexité des processus psycho-sociaux, restera d'un enseignement précieux pour les générations futures.

Au cours de mes séjours au Département de Psychologie Sociale de la PUC, j’ ai pu accompagner Sílvia Lane dans ses activités de directrice de recherche. Elle fut une animatrice intellectuelle dont le dynamisme a encouragé bien des vocations et soutenu bien des talents. Je pense, bien sûr, à ceux que je connais comme Bader Sawaia, Wanderley Codo, Antonio Ciampa qui lui gardent un attachement infaillible. Mais il y en a d'autres qui ont bénéficié de sa patience attentive, de son engagement enthousiaste et de sa participation totale à leurs intérêts. En cela elle fut une authentique pédagogue, épousant les vues de ses élèves tout en sachant les orienter dans le sens d'une psychologie sociale humaniste et éthiquement responsable.

Il ne m'a pas été donné de suivre les travaux de Sílvia Lane dans le champ de la psychologie communautaire, mais il est aisé de voir que c'est dans ce domaine qu'elle a pu le mieux mettre en oeuvre les perspectives développées dès ses premières publications sur la psychologie sociale. En atteste le retentissement de ses écrits dans les travaux de ses disciples et dans les milieux de la recherche au-delà des frontières brésiliennes. Communautaire, elle le fut aussi dans sa pratique scientifique.

Ce sens du partage et de la coopération, servi par un style heureux et toujours inspiré, sinon lyrique, en fait un auteur de référence, digne de figurer parmi les classiques de la psychologie sociale. Indifférente aux modes qui se révèlent vite caduques, Sílvia Lane a su rester fidèle à ses premières options théoriques et pratiques, maintenir à son plus haut niveau le flambeau d'une psychologie sociale critique et engagée. Elle a dû parfois en payer chèrement le prix. Mais son esprit de résistance était resté intact. Il demeurera dans ses oeuvres comme un modèle à suivre.

Denise Jodelet é Doutora em Ciências Sociais pela L'Ecole des Hautes Etudes en Sciences Sociales (EHESS), França. Endereço para correspondência: EHESS Laboratoire de Psychologie Sociale, 105, Boulevard Raspail Montparnasse, Paris, França, 75006. denise.jodelet@wanadoo.fr

La Grande Dame de la Psychologie Sociale

Denise Jodelet

Recebido: 30/07/2007

Aceite final: 14/09/2007

$1^{a}$ revisão: $30 / 07 / 2007$ 